\title{
Clara Schumann \#digital 40 Jahre Frau und Musik und der Start in die Digitalisierung
}

Elisabeth Treydte, Frankfurt a. M.

DOI: $10.25366 / 2020.94$

Zitation: Elisabeth Treydte, "Clara Schumann \#digital. 40 Jahre Frau und Musik und der Start in die Digitalisierung", in: Brückenschläge zwischen Musikwissenschaft und Informatik. Theoretische und praktische Aspekte der Kooperation, in Verbindung mit der Fachgruppe Digitale Musikwissenschaft hrsg. von Stefanie Acquavella-Rauch, Andreas Münzmay und Joachim Veit (= Musikwissenschaft: Aktuelle Perspektiven. Bericht über die Jahrestagung der Gesellschaft für Musikforschung 2019 in Paderborn und Detmold, Bd. 3), Detmold, Musikwissenschaftliches Seminar der Universität Paderborn und der Hochschule für Musik Detmold, 2020, S. 53-57, DOI: 10.25366/2020.94

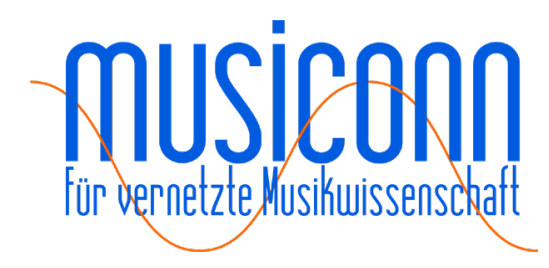




\title{
Clara Schumann \#digital
}

\section{Jahre Archiv Frau und Musik und der Start in die Digitalisierung}

\author{
ELISABETH TREYDTE, FRANKFURT A. M.
}

Das Jahr 2019 war ein ganz besonderes: Nicht nur Clara Schumann konnte ihren Geburtstag feiern, sondern auch das Frankfurter Archiv Frau und Musik. Seit vierzig Jahren schon engagiert es sich für die Sichtbarkeit von Frauen im Musikbetrieb und regt Veranstalter*innen zur paritätischen Ausgestaltung von Konzertprogramm an - und trägt seit all dieser Zeit dazu bei, Komponistinnen wie Clara Schumann u. v. a. bekannt zu machen. Es ist das erklärte Ziel des Trägervereins - dem Internationalen Arbeitskreis Frau und Musik e.V. (IAK) -, das reichhaltige kreative Schaffen von Komponistinnen, Dirigentinnen und Instrumentalistinnen für die Musikpraxis und -forschung zur Verfügung zu stellen. Dafür werden die Bestände sowohl analog als auch teilweise digital archiviert und Einblicke in das Material sind auf vielfältigem Wege sowie stets zugeschnitten auf das individuelle Anliegen leicht möglich.

Zu Zeiten der Archivgründung war von der Digitalisierung noch nichts zu spüren: 1977 erschien ein Artikel in der feministischen Zeitschrift EMMA, der unter dem Titel "Vergessene Komponistinnen"1 die Frage nach dem Verbleib musikschaffender Frauen der vergangenen Jahrhunderte stellte und Musikerinnen, Komponistinnen und Musikwissenschaftlerinnen aufrief, sich dem Vorhaben, die weibliche Seite der Musikgeschichte zu entdecken, anzuschließen. Es folgten kurz darauf erste Treffen, engagierte Frauen diskutierten und tauschten sich aus, suchten in Bibliotheken und Archiven nach der noch unbekannten Musik von Komponistinnen und führten diese auf.

Alsbald gründeten die Frauen den Verein "Internationaler Arbeitskreis Frau und Musik e.V.", der es sich zur Aufgabe machte, "die Kompositionen von Frauen in Vergangenheit und Gegenwart ausfindig zu machen, zu sammeln und aufzuführen und sie damit einer breiten Öffentlichkeit zugänglich zu machen". ${ }^{2}$ Nach und nach entstand eine umfangreiche Sammlung an Noten von Komponistinnen und Dokumenten rund um die Frauenmusikgeschichte, aus der das Archiv Frau und Musik erwachsen ist. 2019, im 40. Jahr seines Bestehens, kann das Archiv Werke von mehr als 1900 Komponistinnen in seinen Beständen nachweisen. Die Sammlung umfasst rund 26.500 Medieneinheiten - Partituren, Bücher, Zeitschriften, Audio-, Videomate-

1 Elke Mascha Blankenburg, "Vergessene Komponistinnen”, in: EMMA 11 (1977), S. 44-46, <https://www.emma.de/ lesesaal/45142\#pages/64> (24.06.2020).

2 <https://www.archiv-frau-musik.de/uber-das-archiv/satzung-internationaler-arbeitskreis-frau-und-musik-ev-2013> (24.06.2020). 
rialien und seltene Drucke sowie Brief- und Notenautographe. Zu den besonderen Schätzen gehört neben originalen Briefen Clara Schumanns eine Postkartensammlung zum Phänomen der Damenblaskapellen um $1900 .^{3}$

Die Digitalisierung der Bestände spielt insbesondere seit 2018 eine große Rolle für das Archiv. Die digitale Erschließung wurde durch drei Drittmittelprojekte möglich, die allesamt beim Digitalen Deutschen Frauenarchiv (DDF) angesiedelt sind. ${ }^{4}$ Das DDF ist eine Plattform, auf der Digitalisate, Essays und Netzwerkkarten rund um die Akteurinnen der Frauenbewegungen im deutschsprachigen Raum zur Verfügung gestellt werden. Eine Vielzahl der in Deutschland vertretenen Lesben-/Frauenarchive, -bibliotheken und -dokumentationsstellen präsentiert auf diesem Portal ihre Bestände und ermöglicht so Einsichten in einzelne lokale Ereignisse sowie in große Bewegungen und Initiativen. Das Archiv Frau und Musik ist seit Beginn des Portals als einziges Musikarchiv mit einer digitalen Sammlung seiner Bestände vertreten. Die digitalisierten Medien finden nach und nach Eingang in den META-Recherchesystem der Plattform und sind somit über die Web-Seite des DDF online zugänglich.

Das erste Projektjahr hatte mit dem Projekt "PARFUMO: Projekt Archiv Frau und Musik Online" das Ziel, einen Teil der Bestände in einer Art digitalen Ausstellung der Öffentlichkeit zur Verfügung zu stellen. ${ }^{5}$ Hierfür wurden zahlreiche Dokumente wie Noten, Schriftgut, Plakate und Bilder, aber auch Audio- und Videomaterialien sowie die von 1979 bis 2015 vom Internationalen Arbeitskreis Frau und Musik herausgegebene Zeitschrift VivaVoce digitalisiert. Darüber hinaus wurden ausgewählte Exponate wie u. a. die selbstgebauten Musikinstrumente der Komponistin Felicitas Kukuck fotografiert. Auf diesem Wege konnte über das DDF-Protal einem breiten Publikum die Vielfalt der Musikgeschichte und ihrer Protagonist*innen veranschaulicht werden. Dies wird durch wissenschaftliche Essays unterstützt, die beispielsweise auf die beruflichen Möglichkeiten von Frauen im (historischen) Musikbetrieb und ihre Netzwerke eingehen. Auch ein biographischer Text über Elke Mascha Blankenburg, Dirigentin und Gründerin des IAK Frau und Musik, ist auf der Plattform des DDF auffindbar. ${ }^{6}$

2019 widmete sich das Archiv Frau und Musik einem Oral-History-Projekt, um die wechselhafte Geschichte der Frauenmusikbewegung zu dokumentieren: Mit dem Titel "MASCHA: Musik-Akteurinnen schaffen Aufmerksamkeit" werden auf der Seite des DDF-Portals mehrere Videointerviews mit Vertreterinnen der Frauen-Musik-Bewegung seit den 1970er Jahren präsentiert, ${ }^{7}$ in denen ihr Einsatz zur Förderung von Frauen im Musikbetrieb, berufliche Werdegänge, Hürden für die Karrieren und Einschätzungen zu den Errungenschaften der Frauen-Musik-Bewegung sowie Wünschen für die weitere Entwicklung zur Gleichstellung von Frauen zur

3 <https://www.archiv-frau-musik.de/bestandsueberblick> (24.06.2020).

4 Vgl. <https://www.digitales-deutsches-frauenarchiv.de> (25.06.2020).

5 Vgl. <https://www.archiv-frau-musik.de/parfumo> (25.06.2020).

6 Anne-Marie Bernhard und Susanne Wosnitzka, "Elke Mascha Blankenburg”, in: Digitales Deutsches Frauenarchiv, <https://www.digitales-deutsches-frauenarchiv.de/akteurinnen/elke-mascha-blankenburg> (24.06.2020).

7 Vgl. <https://www.archiv-frau-musik.de/mascha> (25.06.2020). 
Sprache kommen. Die acht Gespräche werden jeweils durch kurze biografische Personenessays begleitet, um die Geschichte zu kontextualisieren und einen einfachen Zugang zu den Werdegängen der Komponistinnen, Wissenschaftlerinnen und Verlegerinnen zu ermöglichen.

Im Jahr 2020 steht schließlich die Erschließung und Digitalisierung von vier ausgewählten Nachlässen im Vordergrund, mit Hilfe derer Materialien der Komponistinnen Felicitas Kukuck, Leni Alexander, Silvia Alvarez de la Fuente und Weronika Aleksandra Markiewicz gesichert werden können. Außerdem wird durch die Zugriffsmöglichkeiten über das DDF ein weiterer Einblick in für viele noch unbekannte Facetten der Musikgeschichte ermöglicht und somit zu einer sichtbaren Hinterfragung des europäischen Kanons beitragen. Passend dazu und in Anlehnung an ein Chorwerk von Felicitas Kuckuck trägt das Projekt den Titel "Worauf warten wir?".

Die Digitalisierung trägt nicht nur dazu bei, Bestände des Archives zu sichern und einer Expert*innengemeinschaft zugänglich zu machen, sondern öffnet durch die einfachen Zugriffsmöglichkeiten die Inhalte auch für fachfremde Interessierte. Darüber hinaus eignen sich die verschiedenen medialen Formate (Bild, Video, Text) zugleich für die Verwendung in Schule und Universität, sodass Musikgeschichte als eine Vielfalt von Erzählungen, Zugangs-und Entfaltungsmöglichkeiten vermittelt werden kann.

Das Archiv bildete seit seiner Gründung eine Schnittstelle zwischen Musikwissenschaft und Musikpraxis und verknüpft bis heute diese Aspekte in seiner alltäglichen Arbeit. Eingehende Rechercheanfragen betreffen die Suche nach musikalischem Repertoire und musikhistorischen Quellen und mit Beratungsgesprächen werden Konzert- sowie Forschungsvorhaben unterstützt. Darüber hinaus lädt das Archiv selbst regelmäßig zu Musikveranstaltungen, Lesungen oder Fachtagungen ein.

Die Digitalisierung macht es möglich, Quellen auch über größere Distanz zur Verfügung zu stellen. Dass diese für alle Interessierten von großem Wert ist, hat sich ganz besonders in den vergangenen Monaten der Corona-Pandemie gezeigt und bestätigt das Engagement des Archivs Frau und Musik, die weitere Digitalisierung auch in Zukunft anzustreben.

Zitation: Elisabeth Treydte, "Clara Schumann \#digital. 40 Jahre Frau und Musik und der Start in die Digitalisierung", in: Brückenschläge zwischen Musikwissenschaft und Informatik. Theoretische und praktische Aspekte der Kooperation, in Verbindung mit der Fachgruppe Digitale Musikwissenschaft hrsg. von Stefanie Acquavella-Rauch, Andreas Münzmay und Joachim Veit (= Musikwissenschaft: Aktuelle Perspektiven. Bericht über die Jahrestagung der Gesellschaft für Musikforschung 2019 in Paderborn und Detmold, Bd. 3), Detmold, Musikwissenschaftliches Seminar der Universität Paderborn und der Hochschule für Musik Detmold, 2020, S. 53-57, DOI: 10.25366/2020.94

8 Vgl. https://www.archiv-frau-musik.de/woraufwartenwir (25.06.2020). 


\section{Abstract}

The Archives of Women in Music based in Frankfurt a. M. (Germany) was founded in 1979. Its goals are increasing the visibility of women in music, achieving programming parity and making the wealth of creative work by women in music available for performance and research. The Archives assure long-term safe storage of both analogue and digital archive and library content. During the last three years it focused on two collection digitization projects and an oral history project.

\section{Kurzvita}

Elisabeth Treydte, seit 2019 wissenschaftliche Mitarbeiterin im Archiv Frau und Musik / Frankfurt a. M. mit dem Projekt "Wir geben den Ton an! Chancengleichheit für Komponistinnen*” (gefördert von der Mariann-Steegmann-Foundation). Studium der Musikwissenschaft, Germanistik und Romanistik in Frankfurt a. M. und Wien. 2014-2020 wissenschaftliche Mitarbeiterin an der Hochschule für Musik und Theater Hamburg. Promotion zur geschlechterspezifischen Praxeologie zeitgenössischer Komponist*innen in Arbeit. 
Major activities of the Archiv Frau und Musik and its governing association, the International Workgroup on Women in Music, in the $40^{\text {th }}$ year of its existence

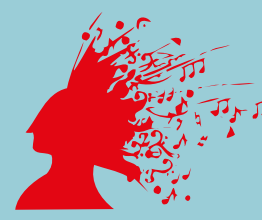

\section{Goals}

Advocacy Increasing the visibility of women in music, achieving programming parity

HerStory Making the wealth of creative work by women in music available for performance and research

Cultural Heritage Assuring long-term safe storage of both analogue and digital archive/library content

\section{History}

1977 Article about forgotten women composers in the "EMMA" magazine

1979 Founding of the Archives in Cologne

1979-2015

Publication of periodical "VivaVoce" 2017 Close cooperation with the (formerly "Info") with articles and news about women in music

1989 Archives move to Kassel
2001 Archives move to Frankfurt am Main

2013 Archives lose part of their funding and are put on the "red list" of endangered archives id.a. Umbrella Organisation of German Lesbian and Women's Archives and the new German Digital Women's Archive (DDF)

\section{Contacts}

Archiv Frau und Musik

Heinrich-Hoffmann-Straße 3

60528 Frankfurt am Main, Germany

Chairwoman Mary Ellen Kitchens M.A.

Associated Academic Researcher Dr. des. Marleen Hoffmann info@archiv-frau-musik.de

Tel.: +49 (0) 69-95928685 | Fax: +49 (0) 69-95928690

www.archiv-frau-musik.de

facebook.com/ArchivFrauUndMusik/

twitter.com/archivfraumusik

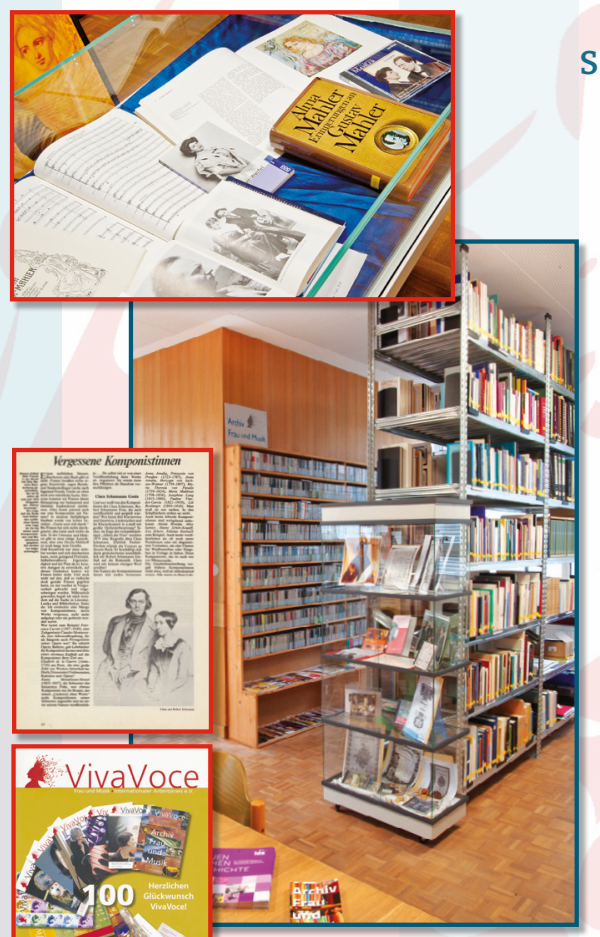

Archive Team

Archive Board

2 permanent employees (parttime)

2 project employees

2 freelancers, numerous interns

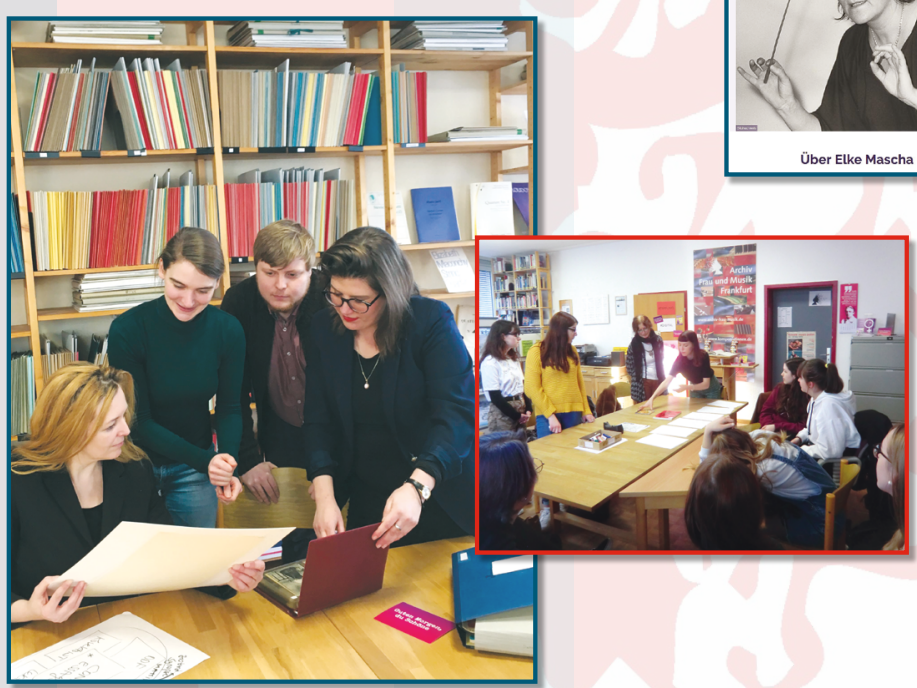

Services and Activities Archiv Frau und Musik

Research services

(repertoire by / information about women composers)

General collection development

(collection focus: musical compositions and legacies of women composers)

Social media monitoring

Collection of press reviews and articles regarding women in music

Creation of annotated repertoire lists of works by women composers

Guided tours of the

Archiv Frau und Musik, in particular for school classes and students

PR and advocacy work /

lobbying for topics related to women in the music field

Networking meetings for women in music organisations throughout Germany

Local, regional, national and international networking efforts

Lectures on related topics

Concerts featuring works

by women composers
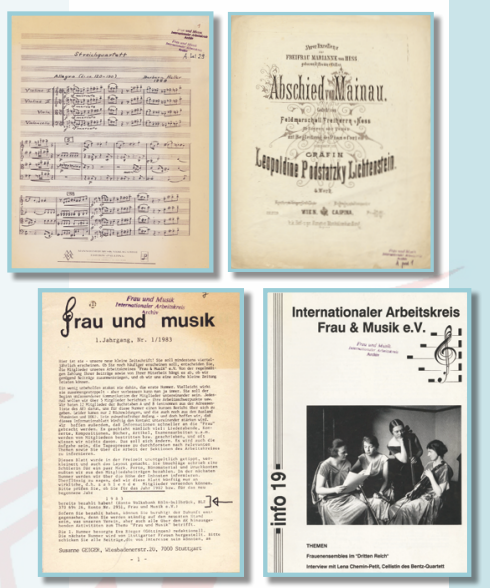

Archival/Library Holdings

Over 26.000 items related to women in music. 1.900 women composers from 52 countries who lived and worked between the $9^{\text {th }}$ century A.D. and the present are represented

About 30 composers have given their manuscripts and documents to the Archives, in particular composers Barbara Heller and Felicitas Kukuck as well as conductor Elke Mascha Blankenburg

\section{Archiv Frau und Musik \\ - Relaunch of the Archive's Webpage (bilingual) at \\ www.archiv-frau-musik.de

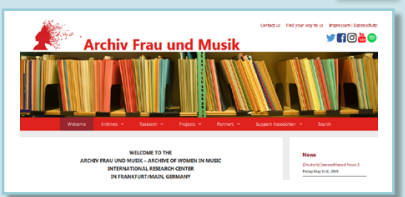

"Setting the Tone for Women in Musicl Equal Opportunity for Women Composers", a 3-year project funded by the Mariann Steegmann Foundation. Advocacy work and networking with universities, yearly focus on one compositional genre (2019: choral music 2020: music pedagogical works, 2021 music for wind and brass instruments) www.archiv-frau-musik.de/ chancengleichheit-fuer-komponistinnen

$4^{\text {th }}$ "Composer in Residence"

Scholarship-awarded to the woman composer Tania Rubio (Mexico).

Three-month residency (July to October, 2019) which includes a response project at a school and a portrait concert in Frankfurt www.archiv-frau-musik.de/en/projekte/ composer-in-residence

- Collection Digitization Project PARFUMO "Projekt Archiv Frau und Musik Online", funded by the Digitales Deutsches Frauenarchiv / Federal Government of Germany. Content presented on the German Digital Women's Archives website: www.deutsches-digitales-frauenarchiv.de

- Oral History Project MASCHA "Musikaktivistinnen schaffen Aufmerksamkeit": Video interviews with central figures in the women's music movement in Germany

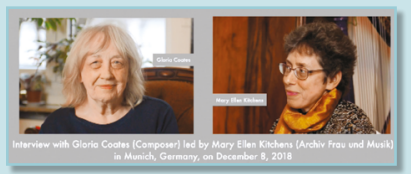

- Migration and Renewal of the Archive's Catalogue Database (FAUST) for improved searching via archive and library portals, for example the META database of the Association of Women's and Lesbian Archives in Germany: www.meta-katalog.eu

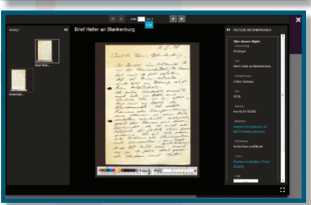

\section{Supporting Institutions}

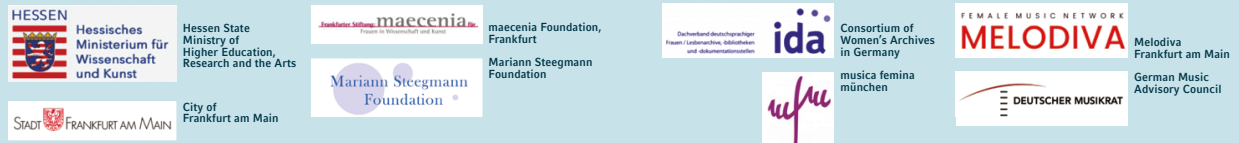

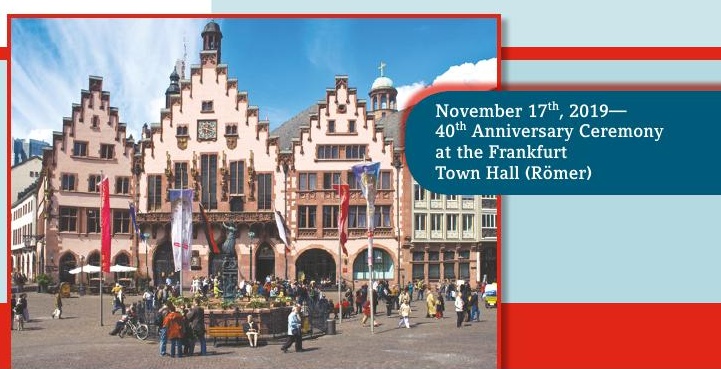




\section{Brückenschläge zwischen Musikwissenschaft und Informatik}

Theoretische und praktische Aspekte der Kooperation

Herausgegeben von Stefanie Acquavella-Rauch,

Andreas Münzmay und Joachim Veit 
Brückenschläge zwischen Musikwissenschaft und Informatik 


\section{Musikwissenschaft: Aktuelle Perspektiven}

Bericht über die Jahrestagung der Gesellschaft für Musikforschung 2019 in Paderborn und Detmold

Herausgegeben von Rebecca Grotjahn und Nina Jaeschke

Band 3 


\section{Brückenschläge zwischen Musikwissenschaft und Informatik}

Theoretische und praktische Aspekte der Kooperation

Beiträge der Symposien zur Digitalen Musikwissenschaft

Osnabrück 2018 und Paderborn 2019

im Rahmen der Jahrestagungen der Gesellschaft für Musikforschung

In Verbindung mit der Fachgruppe Digitale Musikwissenschaft herausgegeben von

Stefanie Acquavella-Rauch, Andreas Münzmay und Joachim Veit

Detmold: Musikwissenschaftliches Seminar der Universität Paderborn und der Hochschule für Musik Detmold 2020 
DOI: $10.25366 / 2020.87$

Online-Version verfügbar unter der Lizenz: Urheberrecht 1.0, $<$ https://rightsstatements.org/page/InC/1.0/?language =de >

Bibliografische Information der Deutschen Nationalbibliothek

Die Deutsche Nationalbibliothek verzeichnet diese Publikation in der Deutschen Nationalbibliografie; detaillierte bibliografische Daten sind im Internet über http://dnb.d-nb.de abrufbar.

\section{Impressum}

Redaktion: Stefanie Acquavella-Rauch, Andreas Münzmay und Joachim Veit Satz: Nina Jaeschke und Joachim Veit

(C) Musikwissenschaftliches Seminar der Universität Paderborn und der Hochschule für Musik Detmold 2020 


\section{INHALT}

Rebecca Grotjahn, Nina Jaeschke

Vorwort zu Band 1-3

IX

Stefanie Acquavella-Rauch, Andreas Münzmay, Joachim Veit

Brückenschläge zwischen Musikwissenschaft und Informatik - Vorbemerkung

$\mathrm{XI}$

\section{KOLLABORATIONEN - KO-LABORATORIEN}

\section{Reinhard Keil}

Der Computer als Denkzeug für hermeneutische Arbeit

\section{Ulrich Konrad}

Philologie und Digitalität. Perspektiven für die Musikwissenschaft im Kontext fächerübergreifender Institutionen

\section{Gudrun Oevel}

Infrastruktureinrichtungen in Forschungsprojekten - Spagat oder Chance?

\section{Dennis Ried}

Erhebung, Transformation und Präsentation digitaler Forschungsdaten

Anna Neovesky, Frederic von Vlahovits

IncipitSearch - Leitfaden zur Zusammenarbeit

Elisabeth Treydte

Clara Schumann \#digital. 40 Jahre Archiv Frau und Musik und der Start in die Digitalisierung

\section{TEXT/DATEN/PROZESSE}

\section{Christine Siegert}

Komponisten-Gesamtausgaben im digitalen Zeitalter: Perspektiven

und Reflexionen am Beispiel Ludwig van Beethovens

Markus Neuwirth, Johannes Hentschel, Martin Rohrmeier

Perspectives of Musical Corpus Studies: The Annotated Mozart Sonatas

Agnes Amminger, Franz Kelnreiter

Leopold Mozarts „Gründliche Violinschule". Zur Textcodierung und -präsentation einer digitalen Edition

\section{Oleksii Sapov}

Algorithmische Automatisierung komplexer Notationsregeln in MEI-XML am Beispiel von Versetzungszeichen 


\section{Susanne Cox, Richard Sänger}

Digitale Fassungsvergleiche am Beispiel von Beethovens Eigenbearbeitungen

Agnes Seipelt

Digitale Edition und Harmonische Analyse mit MEI von Anton Bruckners

Studienbuch

Stefanie Acquavella-Rauch

Musikalische Schaffensprozesse 2.0 - Inkorporation audiovisueller Medien

der populären Musik in Methoden der digitalen Edition

\section{DIGITAL(ISIERT)E MATERIALITÄTEN}

\section{Miriam Akkermann}

(Musik)Instrument (im) Computer

\section{Daniel Fütterer}

Herausforderungen bei der Kodierung von Paratext am Beispiel Neuer Musik mit Live-Elektronik

\section{Matthias Pasdzierny}

How much is the glitch? Das digitale Paradigma als Herausforderung

und Chance für die historische Musikwissenschaft

\section{Shintaro Miyazaki}

Musik für Maschinen?! - Wo sich die Wissenschaft der Medien, des Computers und der Musik treffen und wie sie zusammenarbeiten könnten

\section{MUSIKGESCHICHTE(N) IM NETZ}

\section{Matthias Tischer}

Musikgeschichte der DDR: Ein Pilotprojekt zur digitalen Musikvermittlung

Annette van Dyck-Hemming, Jan Eberhardt, Melanie Wald-Fuhrmann

Ansätze zur Analyse historischer Netzwerke mit Neo4j® - Aus der Projekt-Werkstatt der Datenbank zur Fachgeschichte der Musikwissenschaft

Axel Beer, Martin Bierwisch, Kristina Krämer

Das MMM2 - Ein regionalgeschichtliches Onlinelexikon der Arbeitsgemeinschaft für mittelrheinische Musikgeschichte

\section{Matej Santi}

Was erzählt Fritz Kreislers Geige?

\section{Elias Berner}

Alle Menschen werden Brüder?! Ein historisches Dokument aus dem

Nationalsozialismus in den sozialen Medien 
Gabriele Buschmeier in memoriam 